\title{
MPC for uncertain systems using the Youla parameterizations
}

\author{
Thomsen, Sven Creutz; Niemann, Hans Henrik; Poulsen, Niels Kjølstad
}

Published in:

Proceedings of the 48th IEEE Conference on Decision and Control

Link to article, DOI:

10.1109/CDC.2008.4738799

Publication date:

2008

Document Version

Publisher's PDF, also known as Version of record

Link back to DTU Orbit

Citation (APA):

Thomsen, S. C., Niemann, H. H., \& Poulsen, N. K. (2008). MPC for uncertain systems using the Youla parameterizations. In Proceedings of the 48th IEEE Conference on Decision and Control (pp. 3421-3426). IEEE. https://doi.org/10.1109/CDC.2008.4738799

\section{General rights}

Copyright and moral rights for the publications made accessible in the public portal are retained by the authors and/or other copyright owners and it is a condition of accessing publications that users recognise and abide by the legal requirements associated with these rights.

- Users may download and print one copy of any publication from the public portal for the purpose of private study or research.

- You may not further distribute the material or use it for any profit-making activity or commercial gain

- You may freely distribute the URL identifying the publication in the public portal 


\title{
MPC for uncertain systems using the Youla parameterizations
}

\author{
Sven Creutz Thomsen, Henrik Niemann and Niels Kjølstad Poulsen
}

\begin{abstract}
Several approaches have been taken in the past to deal with uncertainty in constrained predictive control. The major drawbacks of these efforts are usually either conservativeness and/or on-line computational complexity. In this work we examine the possibility of dealing with uncertainty through the use of the primary and the dual Youla parameterizations. The dual Youla parameter can be seen as a frequency weighted measure of the uncertainty and the primary Youla parameter can be seen as a controller for this uncertainty. The work is an application of the methodology in [12] to constraint control.
\end{abstract}

\section{INTRODUCTION}

Model predictive control (MPC) - also commonly denoted constrained predictive control - is a model based control method which has attracted a lot of attention partly due to its popularity in the process industry. The feature which makes it truly innovative is its ability to handle constraints on control action and states/output. This is done through on-line optimization of the future trajectory based on a cost function. The theoretical foundation of nominal linear MPC has matured over the last decades and well established theorems for ensuring stability and feasibility have been established (See eg. [6], [7]).

There has also been established theories for dealing with model uncertainty and disturbances in MPC. These methods are commonly denoted robust MPC (RMPC). Invariant sets have proved effective to deal explicitly with these challenges and still leading to computational tractable problems. This usually leads to optimization problems involving constraints on the form of linear matrix inequalities [3][4][14]. Using this framework it is possible to guarantee (under certain conditions) that the constraints on control actions and states will never be violated. However, there are drawbacks with these methods: The optimization problem, although tractable, can be very complicated compared to basic MPC and therefore typically more computationally expensive. Furthermore, they have a tendency to be overly conservative. Due to the conservative nature of these methods, the trajectory of the system will generally not get very close to the constraints. However, MPC is usually employed in applications where it is attractive to work near the constraints.

We want to avoid the conservative nature of RMPC and still be able to deal with the uncertainties in some sense. In this work we therefore consider a framework with which to handle uncertainty through identification of the unmodeled

S.C. Thomsen and N.K. Poulsen are with the department of Informatics and Mathematical Modeling, Technical University of Denmark, DK-2800 Kgs. Lyngby, Denmark $\{\mathrm{sct}, \mathrm{nkp}\} @ \mathrm{imm} . \mathrm{dtu} . \mathrm{dk}$

H. Niemann is with the department of Electrical Engineering, Technical University of Denmark, DK-2800 Kgs. Lyngby, Denmark hhn@elektro.dtu.dk dynamics. More specifically we identify the dual Youla parameter which can be considered a frequency weighted measure of the uncertainty. We then use the inherent relationship between the dual Youla parameter and primary Youla parameter to design an MPC controller with the objectives of both reducing sensitivity towards the uncertainty and obeying constraints. The idea of using the relationship between the primary and the dual Youla parameter in controller design has been used to design performance enhancing controllers for uncertain plants in [12]. The contribution of the work in this paper is an extension so that it can be incorporated in a natural way in constrained predictive control.

The Youla parameter has previously been used to design MPC controllers with reduced sensitivity towards disturbances in [9][10]. Since sensitivity toward disturbances is reduced the predictions are believed to be more reliable during constraint control. Although some of the same principles are used, it is stressed that the framework in [9][10] has little resemblance to the framework derived in this paper.

\section{NOTATION}

We make use of the following matrix notation: $I_{N \times N}$ denotes the $N$-dimensional identity matrix. $I_{N}$ denotes an $N$-dimensional column vector with ones. $\otimes$ denotes the Kronecker product. We will use the following short notation for the extended observability matrix and Toeplitz matrix:

$$
\mathcal{O}^{N}(A, C)=\left[\begin{array}{lllll}
C^{T} & (C A)^{T} & \left(C A^{2}\right)^{T} & \cdots & \left(C A^{N}\right)^{T}
\end{array}\right]^{T}
$$

$$
\begin{aligned}
& \mathcal{T}^{N}(A, B, C, D)= \\
& {\left[\begin{array}{ccccc}
D & 0 & \cdots & 0 & 0 \\
C B & D & \cdots & 0 & 0 \\
C A B & C B & \cdots & 0 & 0 \\
\vdots & \vdots & \ddots & \vdots & \vdots \\
C A^{N-1} B & C A^{N-2} B & \cdots & C B & D
\end{array}\right]}
\end{aligned}
$$

The notation $\|x\|_{W}^{2}$ is used to denote the weighted 2-norm of a vector $x$ ie.

$$
\|x\|_{W}^{2}=x^{T} W x
$$

\section{SETUP AND PRELIMINARIES}

\section{A. System setup}

We consider the following linear discrete time-invariant system:

$$
\Sigma=\left(\begin{array}{cc}
G_{q p} & G_{q u} \\
G_{y p} & G_{y u}
\end{array}\right)
$$


$u_{k} \in \mathbb{R}^{n_{u}}$ is the control input. $p_{k} \in \mathbb{R}^{n_{p}}$ is the disturbance input. $q_{k} \in \mathbb{R}^{n_{q}}$ is an auxiliary output. $y_{k} \in \mathbb{R}^{n_{y}}$ is a measurable output. The uncertainty enters the system through the relation

$$
p_{k}=\Delta q_{k}
$$

where $\Delta$ is an unknown LTI perturbation. The system is assumed controlled by the controller $K$

$$
u_{k}=K y_{k}
$$

It is assumed that $K$ has been designed such that the system is robustly stable for

$$
\|\Delta\|_{\infty}=\sup _{|z|=1} \sigma_{\max }(\Delta(z)) \leq 1
$$

where $\sigma_{\max }$ is the maximum singular value. The setup is illustrated in Fig. 1. The auxiliary signal $v_{k} \in \mathbb{R}^{n_{u}}$ shown in the figure will be used to avoid constraint violation.

\section{B. Model predictive control}

Model predictive control (MPC) [6] or constrained predictive control is a receding horizon methodology where an optimization problem is solved at every sample time $k$. We introduce MPC in the context of the prestabilized system in Fig. 1. The basic idea is to find the control sequence $V_{k}=\left\{v_{k}, v_{k+1}, \ldots, v_{k+N}\right\}$ which minimizes a finite horizon cost. One then uses the first element of $V_{k}$ as the control action. In the nominal case $\Delta=0$ the cost is commonly a quadratic cost on output $y_{k}$ and input $v_{k}$

$$
J_{k}=\sum_{i=k}^{N+k}\left\|y_{i}\right\|_{W_{y}}^{2}+\left\|v_{i}\right\|_{W_{v}}^{2}
$$

where $W_{y} \geq$ and $W_{v}>0$ are suitable weighting matrices. Linear constraints on output and input are usually included and can be written on the form

$$
\begin{aligned}
P_{y} y_{i+1} & \leq I_{n_{c y}} \quad k \leq i \leq N-1 \\
P_{u} u_{i} & \leq I_{n_{c u}}
\end{aligned}
$$

over the finite control horizon. The $n_{c y}$ and $n_{c u}$ denotes the number of output and input constraints respectively.

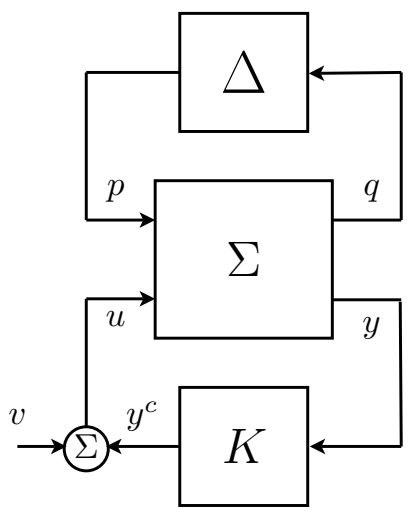

Fig. 1: System setup: The system is controlled by the feedback controller $K$ which stabilizes $\Sigma$ subject to the unknown LTI system $\Delta$.
The basic optimization problem is easily written explicitly as a problem in the control sequence $V_{k}$ ie. as a static optimization problem. The problem with constraints can then be solved using a quadratic programming (QP) solver.

\section{The Youla parameterizations}

The Youla parameterization of all stabilizing controllers is well known and has been used to large extend in controller synthesis (See eg. [13]). With reference to Fig. 1 we consider the system $G \equiv G_{y u}$ and stabilizing controller $K$ (both transfer matrices). System and controller can be written as left or right co-prime factorizations:

$$
\begin{aligned}
G & =N_{r} M_{r}^{-1}=M_{l}^{-1} N_{l} \\
K & =U_{r} V_{r}^{-1}=V_{l}^{-1} U_{l}
\end{aligned}
$$

where $N_{r}, M_{r}, U_{r}, V_{r}, N_{l}, M_{l}, U_{l}, V_{l} \in R H_{\infty}$ and satisfy the double Bezout identity

$$
\begin{aligned}
\left(\begin{array}{ll}
I & 0 \\
0 & I
\end{array}\right) & =\left(\begin{array}{cc}
V_{l} & -U_{l} \\
-N_{l} & M_{l}
\end{array}\right)\left(\begin{array}{cc}
M_{r} & U_{r} \\
N_{r} & V_{r}
\end{array}\right) \\
& =\left(\begin{array}{cc}
M_{r} & U_{r} \\
N_{r} & V_{r}
\end{array}\right)\left(\begin{array}{cc}
V_{l} & -U_{l} \\
-N_{l} & M_{l}
\end{array}\right)
\end{aligned}
$$

Then all controllers which stabilizes $G$ are given as:

$$
\begin{aligned}
& K(Q)=\left(U_{r}+M_{r} Q\right)\left(V_{r}+N_{r} Q\right)^{-1} \\
& K(Q)=\left(V_{l}+Q N_{l}\right)^{-1}\left(U_{l}+Q M_{l}\right)
\end{aligned}
$$

where $Q \in R H_{\infty}$ is called the Youla parameter.

The dual of the Youla parameterization is all systems stabilized by a given controller [8][12]. This is commonly denoted the dual Youla parameterization. The parameterization can be written as follows:

$$
\begin{aligned}
& G(S)=\left(N_{r}+V_{r} S\right)\left(M_{r}+U_{r} S\right)^{-1} \\
& G(S)=\left(M_{l}+S U_{l}\right)^{-1}\left(N_{l}+S V_{l}\right)
\end{aligned}
$$

where $S \in R H_{\infty}$ is the dual Youla parameter. The nominal system $G$ is naturally attained for $S=0$. A useful interpretation of $S$ is that of a frequency shaped version of the uncertainty [12]. Provided that $K$ robustly stabilizes $G$ there exist a map between $\Delta$ and $S \in R H_{\infty}$ [8]:

$$
S(\Delta)=T_{3} \Delta\left(I-T_{1} \Delta\right)^{-1} T_{2}
$$

where

$$
\begin{gathered}
T_{1}=G_{q p}+G_{q u} U_{r} M_{l} G_{y p} \\
T_{2}=G_{q u} M_{r}, T_{3}=M_{l} G_{y p}
\end{gathered}
$$

An interesting property of the parameterization is that the Youla parameter $Q$ looks directly into the dual Youla parameter $S$ [8][12]:

$$
\epsilon_{k}=S \eta_{k}
$$

where $\epsilon_{k}$ and $\eta_{k}$ are the input and output of Q respectively. One can therefore think of $Q$ as a controller for the dual Youla parameter $S$. This also has the interpretation of $Q$ controlling the model uncertainty. Actually, it turns out that the pair $(G(S), K(Q))$ is stabilizing if and only if the pair $(S, Q)$ is stabilizing. The work in [12] is devoted to exploiting this principle. 


\section{METHOD}

The main observation which we will take advantage of in MPC is the fact that the dual Youla parameter $S$ can be regarded as the uncertainty of the system. Hence, if the performance of the system is unsatisfactory (eg. if constraints are violated), we can use an identification scheme to gain information about $S$ and make actions accordingly ie. reconfigure the controller. Since standard identification procedures exist for solving this problem, we will only briefly cover this problem in the paper.

In constraint predictive control an optimization problem is setup by deriving explicitly how the trajectory of the system evolves over a finite horizon. Hence, in this section we establish how the trajectory of the system depends on the dual Youla parameter $S$. Furthermore, we show how to include the Youla parameter $Q$ in the predictions.

Since the Youla parameterization is non-unique we need to introduce a specific realization. In this work we use an observer form of the Youla parameterization. The realization is shown in Fig. 2 where the observer is based on the nominal system $G \equiv G_{y u}$ (For details see [2]). The realization is only valid for $K \in R H_{\infty}$. All stabilizing controllers for the nominal systems is hence parameterized by $Q \in R H_{\infty}$ with input $\epsilon_{k}$ and output $\eta_{k}$. We have included the auxiliary signal $v_{k}$ which was used as the MPC control signal in section III. Looking at the input-output connection $v_{k}-\epsilon_{k}$ we look directly into the controlled dual Youla parameter $\check{S}$ :

$$
\epsilon_{k}=\check{S} v_{k}=(I-S Q)^{-1} S v_{k}
$$

This follows immediately from the theory presented in section III-C. We will show how this can be used when deriving the predictions for the MPC optimization problem.

\section{A. Predictions in presence of the dual Youla parameter $S$}

We will now derive the prediction equations in the presence of the assumed knowledge about $S=S(\Delta)$. We will for simplicity assume that $Q=0$ for which relation (23) reduces

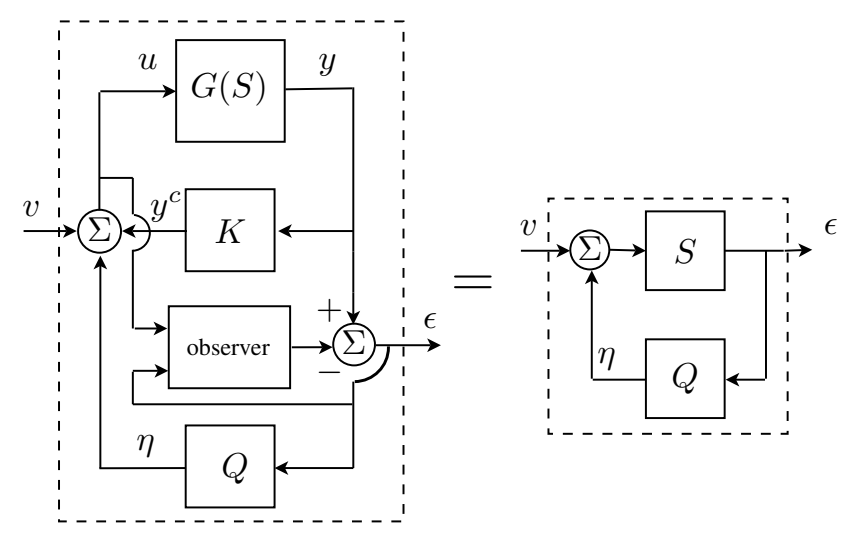

(a) Observer form of Youla parameteri- $\quad$ (b) Equivalent representation zation

Fig. 2: The Youla parameterization is realized in the observer form [2]. It follows from the theory that the dynamic system from $v_{k}$ to $\epsilon_{k}$ is the closed loop connection of $S$ and $Q$. to $\epsilon_{k}=S v_{k}$ Later we show how the result immediately generalizes to the case with non-zero $Q$.

The dynamics of the nominal system $G \equiv G_{y u}$, controller $K$ and observer $G_{o}$ are:

$$
\begin{gathered}
G=\left\{\begin{array}{cc}
x_{k+1}=A x_{k}+B u_{k} \\
y_{k}= & C x_{k}
\end{array}\right. \\
K=\left\{\begin{array}{cc}
x_{k+1}^{c}=A_{c} x_{k}^{c}+B y_{k} \\
y_{k}^{c}=C_{c} x_{k}^{c}+D y_{k}
\end{array}\right. \\
G_{o}=\left\{\begin{array}{cc}
\hat{x}_{k+1}=A \hat{x}_{k}+B u_{k}+L \epsilon_{k} \\
\hat{y}_{k}= & C \hat{x}_{k}
\end{array}\right. \\
u_{k}=v_{k}+y_{k}^{c}, \quad \epsilon_{k}=y_{k}-C \hat{x}_{k}
\end{gathered}
$$

where $x_{k} \in \mathbb{R}^{n_{x}}$ is the state vector of the nominal system, $x_{k}^{c} \in \mathbb{R}^{n_{k}}$ is the state vector of the controller and $\hat{x}_{k} \in \mathbb{R}^{n_{x}}$ is the state vector of the observer. The output injection gain $L$ is naturally chosen such that $(A, C L)$ is a stabilizing pair.

The equations (24)-(26) represents the nominal dynamics $\Delta=0(S(\Delta)=0)$. Disregarding transients caused by disturbances and initial conditions the observer estimation error will be $\epsilon_{k}=0$ no matter what the input sequence $v_{k}$ is. In the presence of uncertainty $\Delta \neq 0(S(\Delta) \neq 0)$ the deterministic dynamic response of $\epsilon_{k}$ given the input $v_{k}$ is determined by the dual Youla parameter $S$. We assume that $S$ has the following state space representation:

$$
S \equiv S(\Delta)=\left\{\begin{array}{c}
x_{k+1}^{s}=A_{s} x_{k}^{s}+B_{s} v_{k} \\
\epsilon_{k}=C_{s} x_{k}^{s}
\end{array}\right.
$$

where $x_{k}^{s} \in \mathbb{R}^{n_{s}}$ is the state vector of $S$. For the sake of simplicity we have assumed that there is no direct term in $S$. Combining equations (25)-(26) we get the following expression governing the evolution of $y_{k}$ and $u_{k}$ :

$$
\begin{aligned}
{\left[\begin{array}{c}
x_{k+1}^{c} \\
\hat{x}_{k+1}
\end{array}\right]=} & \underbrace{\left[\begin{array}{cc}
A_{c} & B_{c} C \\
B C_{c} & A+B D_{c} C
\end{array}\right]}_{\bar{A}}\left[\begin{array}{l}
x_{k}^{c} \\
\hat{x}_{k}
\end{array}\right] \\
& +\underbrace{\left[\begin{array}{c}
0 \\
B
\end{array}\right]}_{\bar{B}} v_{k}+\underbrace{\left[\begin{array}{c}
B_{c} \\
B D_{c}+L
\end{array}\right]}_{H} \epsilon_{k} \\
y_{k}= & \underbrace{\left[\begin{array}{ll}
0 & C
\end{array}\right]}_{\bar{C}_{y}}\left[\begin{array}{l}
x_{k}^{c} \\
\hat{x}_{k}
\end{array}\right]+\epsilon_{k} \\
u_{k}= & \underbrace{\left[\begin{array}{ll}
C_{c} & D_{c} C
\end{array}\right]}_{\bar{C}_{u}}\left[\begin{array}{l}
x_{k}^{c} \\
\hat{x}_{k}
\end{array}\right]+v_{k}+D_{c} \epsilon_{k}
\end{aligned}
$$

The residual $\epsilon_{k}$ has a natural interpretation as a correction term due to the perturbation $S$. Disregarding transients caused by disturbances and initial conditions the deterministic evolution of $y_{k}$ and $u_{k}$ is therefore completely described by equations (29)-(31) and the dual Youla parameter (28).

Remark 1: It is evident from equation (30) that $\epsilon_{k}$ only can be used to correct the predictions of $y_{k}$ and not general linear combinations of $x_{k}$. Therefore, we can only handle constraint on the measurable output $y_{k}$ and not general constraints on the states. 
Remark 2: The states $x_{k}^{s}$ of $S$ are naturally not accessible in general. Therefore, we have to rely on estimated states $\hat{x}_{k}^{s}$ obtained through an observer:

$$
\begin{aligned}
\hat{x}_{k+1}^{s} & =A_{s} \hat{x}_{k}^{s}+B_{s} v_{k}+L_{s}\left(\epsilon_{k}-C_{s} \hat{x}_{k}^{s}\right) \\
\hat{\epsilon}_{k} & =C_{s} \hat{x}_{k}^{s}
\end{aligned}
$$

where $\hat{x}_{k}^{s} \in \mathbb{R}^{n_{s}}$ is the state vector of the dual Youla parameter. Hence, when referring to the state $x_{k}^{s}$ in the following it is implicitly implied that it could be an estimate.

Based on the state $x_{k}^{s}$ of the dual Youla parameter, the observer state $\hat{x}_{k}$ and the state of the controller $x_{k}^{c}$ we can find (through iterations) the future trajectory of $y_{k}, u_{k}$ and $\epsilon_{k}$ given the trajectory of $v_{k}$. Let $Y_{k}, U_{k}, E_{k}, V_{k}$ denote the corresponding stacked vectors of $N$ step trajectories eg.:

$$
Y_{k}=\left[\begin{array}{lllll}
y_{k}^{T} & y_{k+1}^{T} & y_{k+2}^{T} & \cdots & y_{k+N}^{T}
\end{array}\right]^{T}
$$

We can write the future $N$ predictions of $y_{k}, u_{k}$ and $\epsilon_{k}$ as

$$
\begin{aligned}
Y_{k} & =\mathcal{A}_{y} z_{k}+\mathcal{B}_{y} V_{k}+\mathcal{H}_{y} E_{k} \\
U_{k} & =\mathcal{A}_{u} z_{k}+\mathcal{B}_{u} V_{k}+\mathcal{H}_{u} E_{k} \\
E_{k} & =\mathcal{A}_{s} x_{k}^{s}+\mathcal{B}_{s} V_{k} \\
z_{k} & =\left[\begin{array}{ll}
x_{k}^{c T} & \hat{x}_{k}^{T}
\end{array}\right]^{T}
\end{aligned}
$$

where $\mathcal{A}_{y}, \mathcal{B}_{y}, \mathcal{H}_{y}, \mathcal{A}_{u}, \mathcal{B}_{u}, \mathcal{H}_{u}, \mathcal{A}_{s}, \mathcal{B}_{s}$ are defined as the following extended observability and Toeplitz matrices (See definition of $\mathcal{O}^{N}$ and $\mathcal{T}^{N}$ in section II).

$$
\begin{aligned}
\mathcal{A}_{y} & =\mathcal{O}^{N}\left(\bar{A}, \bar{C}_{y}\right), \mathcal{B}_{y}=\mathcal{T}^{N}\left(\bar{A}, \bar{B}, \bar{C}_{y}, 0\right) \\
\mathcal{A}_{u} & =\mathcal{O}^{N}\left(\bar{A}, \bar{C}_{u}\right), \mathcal{B}_{y}=\mathcal{T}^{N}\left(\bar{A}, \bar{B}, \bar{C}_{u}, 0\right) \\
\mathcal{A}_{s} & =\mathcal{O}^{N}\left(A_{s}, C_{s}\right), \mathcal{B}_{s}=\mathcal{T}^{N}\left(A_{s}, B_{s}, C_{s}, 0\right) \\
\mathcal{H}_{y} & =\mathcal{T}^{N}(\bar{A}, H, \bar{B}, I), \mathcal{H}_{u}=\mathcal{T}^{N}\left(\bar{A}, H, \bar{B}, D_{c}\right)
\end{aligned}
$$

The closed form prediction of $Y_{k}$ hence becomes

$$
\begin{aligned}
Y_{k} & =\underbrace{\mathcal{A}_{y} z_{k}+\mathcal{B}_{y} V_{k}}_{\text {Nominal }}+\underbrace{\mathcal{H}_{y}\left(\mathcal{A}_{s} x_{k}^{s}+\mathcal{B}_{s} V_{k}\right)}_{\text {Perturbation }} \\
& =Y_{k}^{(G, K)}+Y_{k}^{S}
\end{aligned}
$$

where $Y_{k}^{(G, K)}$ is the contribution owing to the nominal dynamics and $Y_{k}^{S}$ is the contribution owing to the dual Youla parameter. Likewise the closed loop predictions of $u_{k}$ becomes:

$$
\begin{aligned}
U_{k} & =\underbrace{\mathcal{A}_{u} z_{k}+\mathcal{B}_{u} V_{k}}_{\text {Nominal }}+\underbrace{\mathcal{H}_{u}\left(\mathcal{A}_{s} x_{k}^{s}+\mathcal{B}_{s} V_{k}\right)}_{\text {Perturbation }} \\
& =U_{k}^{(G, K)}+U_{k}^{S}
\end{aligned}
$$

where $U_{k}^{(G, K)}$ is the contribution owing to the nominal dynamics and $U_{k}^{S}$ is the contribution owing to the dual Youla parameter.

Remark 3: A nice property of the derived predictions is the separability into the nominal contribution $(S(\Delta)=0$ ) and the contribution due to $S(\Delta) \neq 0$. Hence, there is no need for total reconfiguration of the MPC controller to take $S$ into account, it should simply be able to take the corrections into account through a plug-in mechanism.
Remark 4: The future knowledge of a reference or setpoint is easily incorporated in the predictions. The important thing to remember is that the reference should be input to the dual Youla parameter.

\section{B. Adding a Youla parameter $Q$ for controlling $S$}

Knowing the perturbation $S \equiv S(\Delta)$ the closed-loop performance can be enhanced by including a Youla parameter $Q$ for controlling $S$. We assume that the Youla parameter has the following state space realization:

$$
Q=\left\{\begin{array}{c}
x_{k+1}^{q}=A_{Q} x_{k}^{q}+B_{Q} \epsilon_{k} \\
\eta_{k}=C_{Q} x_{k}^{q}+D_{Q} \epsilon_{k}
\end{array}\right.
$$

where $x_{k}^{q} \in \mathbb{R}^{n_{q}}$ is the state vector of the Youla parameter. Since $Q$ looks directly into $S$ the corrected predictions will now be made on the basis of the controlled dual Youla parameter:

$$
\check{S}=(I-S Q)^{-1} S
$$

It is straightforward to derive the predictions with the controlled dual Youla parameter. The predictions are simple made using $\check{S}$ with state vector $x_{k}^{\breve{s}}=\left[\begin{array}{ll}x_{k}^{s T} & x_{k}^{q T}\end{array}\right]^{T}$ instead of $S$. The prediction of $y_{k}$ and $u_{k}$ can now be written on the following form over the prediction horizon:

$$
\begin{aligned}
& Y_{k}=Y_{k}^{(G, K)}+Y_{k}^{(S, Q)} \\
& U_{k}=U_{k}^{(G, K)}+U_{k}^{(S, Q)}
\end{aligned}
$$

where the notation $(S, Q)$ has been used to indicate the contribution owing to the controlled dual Youla parameter.

\section{Identification of $S$}

Using well established system identification methods it is possible to identify $S$ using the auxiliary signal $v_{k}$ as excitation signal. So far we have not considered noise, however, in the general noisy case the signal $\epsilon_{k}$ is related to the signal $v_{k}$ through the following equation:

$$
\epsilon_{k}=\check{S} v_{k}+e_{k}
$$

where $e_{k}$ is the noise contribution. With $v_{k}$ persistently exiting and uncorrelated with $e_{k}$ it is possible to get an unbiased estimate of $\check{S}$ using eg. an output-error method [5]. Since $Q$ is user defined $S$ is then easily established from $\breve{S}$ :

$$
S=(I+\check{S} Q)^{-1} \check{S}
$$

For a more rigorous treatment the reader is referred to [1][12].

Remark 5: Identification of $S$ should be done only when the constrained control action is inactive. When the constrained control is active we effectively have an extra nonlinear loop around the system. As will be shown shortly, we setup an MPC strategy where the constrained control action is active only when strictly necessary. 


\section{MPC strategy}

The predictions derived so far can be used to implement a MPC scheme which ensures satisfaction of constraints given the assumed knowledge of $S \equiv S(\Delta)$.

The cost for the MPC controller could in general be given by the cost in equation (8). If $W_{y}>0$ this will give a control signal $v_{k}$ which is active even though no constraints are violated over the prediction horizon. This is unattractive since we already assume that the controller $K$ and the Youla parameter $Q$ have been designed to deliver desired performance. It will basically interfere with the criteria on which the controllers $K$ and $Q$ were designed.

To make sure that the MPC controller only interferes when necessary we set $W_{y}=0$ and the cost reduces to:

$$
J_{k}=\sum_{i=k}^{N}\left\|v_{i}\right\|_{W_{v}}^{2}
$$

We minimize this objective subject to the constraints (9)(10) and the dynamics of the closed loop system consisting of the pair $(G(S), K(Q))$. The deterministic evolution of the trajectory is naturally described by the prediction equations given in the previous sections. Therefore, the constrained optimization problem can in the usual way be written as a constrained static optimization problem in the decision vector $V_{k}$ and solved using a QP solver.

Under certain assumptions the suggested receding horizon control will guarantee stability: There exists a finite horizon $N \in \mathbb{N}$ for which cost (53) is equal to the infinite horizon cost. The infinite horizon problem is guaranteed stable if we know the system perfectly (ie. we have identified $S$ ) and the state estimation errors are sufficiently small. This is a special case of the results in [11] for prestabilized systems. In [11] an algorithm is given for choosing $N$ online.

In practice these assumptions are unlikely to hold, and as is common practice in real applications, we might simply accept that situations could theoretically occur which leads to instability or infeasibility of the optimization problem.

\section{E. Extensions}

The true potential of the setup introduced so far lies in the extensions. The framework provides the basis for using the powerful ideas described in [12] together with constrained control action. We will confine ourself to a short description of one immediate extension possibility: The socalled iterative $(S, Q)$ design. The framework derived so far is actually the first step of the iterative design.

1) Iterative design: After the first $Q=Q_{1}$ has been designed for the identified $S$ the iterative method proceeds as follows: If performance is unacceptable we re-identify the uncertainty. However, this time it is the controlled dual Youla parameter we are looking into:

$$
\check{S}=(I-S Q)^{-1} S
$$

we then simply design an extra controller $Q_{2}$ for dealing with $\check{S}$. The total controller is hence $Q=Q_{1}+Q_{2}$. These

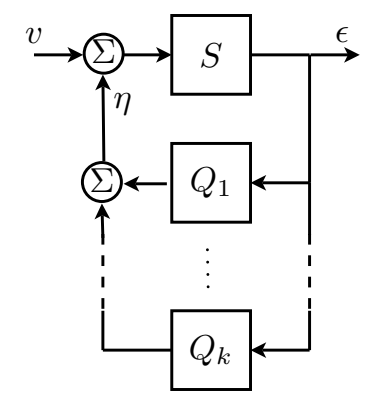

Fig. 3: Visualization of iterative design

steps are repeated until acceptable performance is attained. The design is illustrated in Fig. 3.

After the $k$ th iteration we have the Youla parameter

$$
Q=Q_{1}+Q_{2}+\cdots+Q_{k}
$$

which is the sum of Youla parameters identified at each iteration. $S$ can be derived from the controlled dual Youla parameter $\check{S}$ identified at the $k$ th iteration using equation (52).

The algorithm is easily used together with the setup in section IV due to the modularity of the setup. The predictions of $Y_{k}$ and $U_{k}$ for the $k$ th iteration are simply corrected based on the $k$ th identified (closed-loop) dual Youla parameter and $k$ th Youla parameter.

\section{ILLUSTRATIVE EXAMPLE}

In this section we illustrate the potential performance enhancement when using the presented framework. We consider a two cart system shown in Fig. 4. The left cart (cart 1) represents the nominal dynamics and the cart to the right (cart 2) represents the perturbation. The objective is to regulate the position $x$ of the left cart by applying a force $u$ to the cart. We introduce the regulation constraint:

$$
|x| \leq 1
$$

Introducing the following state vectors

$$
z=\left[\begin{array}{ll}
x & \dot{x}
\end{array}\right]^{T}, \quad z_{\Delta}=\left[\begin{array}{ll}
x_{\Delta} & \dot{x}_{\Delta}
\end{array}\right]^{T}
$$

and putting the system description in the form in Fig. 1 we get the following continuous time description of the nominal

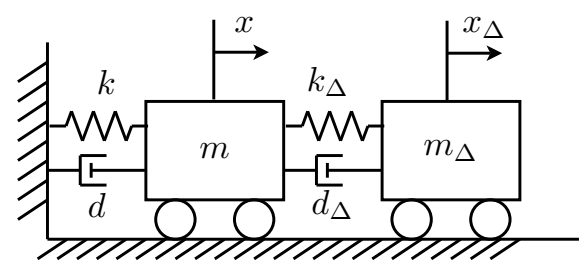

Fig. 4: Sketch of the two cart system 
dynamics:

$$
\Sigma=\left\{\begin{array}{l}
\dot{z}=\left[\begin{array}{cc}
0 & 1 \\
-\frac{k}{m} & -\frac{d}{m}
\end{array}\right] z+\left[\begin{array}{l}
0 \\
1
\end{array}\right] p+\left[\begin{array}{l}
0 \\
\frac{1}{m}
\end{array}\right] u \\
q=z \\
y=z
\end{array}\right.
$$

and the following description of the perturbation:

$$
\Delta=\left\{\begin{array}{l}
\dot{z}_{\Delta}=\left[\begin{array}{cc}
0 & 1 \\
-\frac{k_{\Delta}}{m_{\Delta}} & -\frac{d_{\Delta}}{m_{\Delta}}
\end{array}\right] z_{\Delta}+\left[\begin{array}{cc}
0 & 0 \\
\frac{k_{\Delta}}{m_{\Delta}} & \frac{d_{\Delta}}{m_{\Delta}}
\end{array}\right] q \\
p=\left[\begin{array}{ll}
\frac{k_{\Delta}}{m} & \frac{d_{\Delta}}{m}
\end{array}\right] z_{\Delta}+\left[\begin{array}{cc}
-\frac{k_{\Delta}}{m} & -\frac{d_{\Delta}}{m}
\end{array}\right] q
\end{array}\right.
$$

The chosen parameter values are as follows: $m=1 \mathrm{~kg} . k=$ $1 \mathrm{~N} / \mathrm{m} . d=1 \mathrm{~N} / \mathrm{m} \cdot \mathrm{s} . m_{\Delta}=0.5 \mathrm{~kg} . k_{\Delta}=1 \mathrm{~N} / \mathrm{m} . d_{\Delta}=0.01$ $\mathrm{N} / \mathrm{m} \cdot \mathrm{s}$. For simulation purposes we consider the discretized dynamics of (58)-(59) where the sample time $T_{s}=1.2 \mathrm{~s}$ has been chosen. In the example we will assume perfect knowledge about $S$ which can be found through the relation (19). In practice we would naturally be confined to identify $S$ through an identification scheme as stated in section IV-C. However, this is not the focus of this example. The minimal representation of $S \equiv S(\Delta)$ is a sixth order system.

To illustrate the potential improvements with the presented framework, we increase the complexity of the controller step by step. The following four control configurations are tested: Nominal (robust) feedback controller K (Labeled K1). Additional nominal constraint handling (K2). Additional correction of predictions based on the dual Youla parameter $S$ (K3). Additional Youla parameter $Q$ to control the dual Youla parameter (K4).

The nominal feedback controller is an LQ controller designed for the nominal dynamics ie. the dynamics of cart 1 which provides robust stability in the presence of the perturbation $\Delta$. The state cost is $W_{z}=I_{2 \times 2}$ and the control cost is $W_{u}=10$. The dual Youla parameter $Q$ is designed as an LQG controller based on the dynamics of $S$. The LQ state cost is $W_{x^{s}}=I_{6 \times 6}$ and the LQ control cost is $W_{\eta}=0.1$. The state noise covariance matrix is chosen as $R_{x^{s}}=I_{6 \times 6}$ and the output noise covariance matrix is chosen as $R_{\epsilon}=I_{2 \times 2}$.

The MPC controller is designed for the system as described in section IV-D. The control horizon is chosen to $N=10$ and the control cost is $W_{v}=1$.

In the simulations we step the reference from 0 to 1 . This means that we want the position of cart 1 to end at $x=1$ but without violating the constraint $|x| \leq 1$. The simulations are shown in Fig. 5. The controller K1 does not satisfy the constraint, which is expected since constraint handling is not included in its design criteria. Constraints are still not met with nominal constraint handling due to erroneous predictions (K2). Correcting the predictions (K3) based on the dual Youla parameter constraints are respected. Performance is increased by the addition of the Youla parameter for controlling the dual Youla parameter (K4).

\section{CONCLUSION}

We have presented a framework for taking advantage of the primary and the dual Youla parameter in constrained

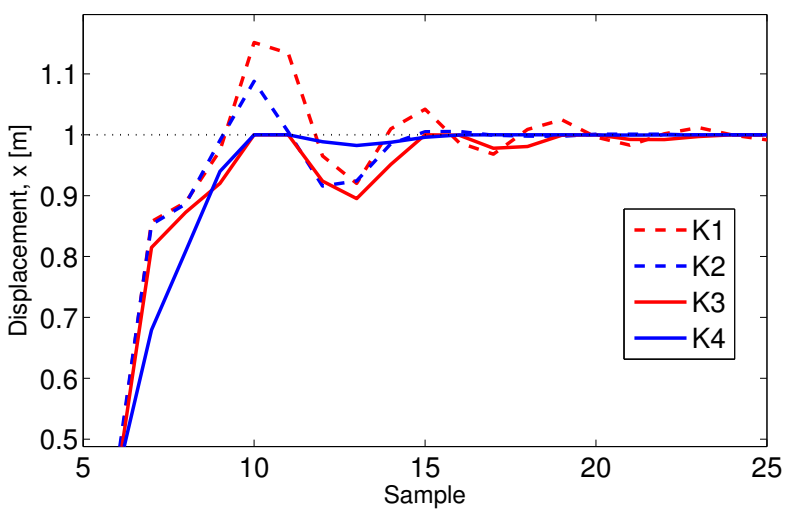

Fig. 5: Simulation with stepped reference

predictive control. Based on a specific realization of the Youla parameterization we derive explicitly the predictions on which the MPC optimization should be made. It is shown that the predictions consist of a nominal contribution owing to the nominal dynamics and a contribution owing to the Youla parameterizations. The MPC problem is formulated such that the MPC controller is active only when there is danger of constraint violation. An example illustrated the potential performance enhancement in using the framework.

\section{REFERENCES}

[1] F. De Bruyne, B. Anderson, and N. Linard. The hansen scheme revisited. Decision and Control, 1998. Proceedings of the 37th IEEE Conference on, 1:706-711, 1998.

[2] J.Y. Ishihara and R.M.R.M. Sales. Doubly coprime factorizations related to any stabilizing controllers in state space. Automatica, 35(9):1573-1577, 1999.

[3] M.V. Kothare, V. Balakrishnan, and M. Morari. Robust constrained model predictive control using linear matrix inequalities. Automatica, 32(10):1361-1379, 1996.

[4] B. Kouvaritakis, J.A. Rossiter, and J. Schuurmans. Efficient robust predictive control. IEEE Transactions on Automatic Control, 45(8):15451549, 2000.

[5] L. Ljung. System Identification: Theory for the User. Prentice Hall, second edition edition, 1999.

[6] J.M. Maciejowski. Predictive control with constraints. Prentice Hall, 2002.

[7] D.Q. Mayne, J.B. Rawlings, C.V. Rao, and P.O.M. Scokaert. Constrained model predictive control: Stability and optimality. Automatica, 36(6):789-814, 2000.

[8] H. Niemann. Dual youla parameterisation. IEE Proceedings-Control Theory and Applications, 150:493-497, 2003.

[9] J.A. Rossiter, L. Chisci, and B. Kouvaritakis. Optimal disturbance rejection via youla parameterisation in constrained lq control. Proceedings of the 6th IEEE Mediterranean Conference. Theory and Practice of Control and Systems, pages 271-6, 1998.

[10] J.A. Rossiter and B. Kouvaritakis. Targetted sensitivity with constrained linear predictive control. Proceedings of the IASTED International Conference Applied Simulation and Modelling, pages 455-60, 2002.

[11] P.O.M. Scokaert and J.B. Rawlings. Constrained linear quadratic regulation. IEEE Transactions on Automatic Control, 43(8):1163$1169,1998$.

[12] T-T. Tay, I. Mareels, and J.B. Moore. High Performance Control. Birkhuser, 1998.

[13] M. Vidyasagar. Control System Synthesis. The M.I.T. Press, 1985.

[14] Z. Wan and M.V. Kothare. An efficient off-line formulation of robust model predictive control using linear matrix inequalities. Automatica, 39:837-846, 2003. 